\title{
Study on the Relationship Between Academic Performance, Neurobehavioral Ability and Personality Characteristics of Pilot cCadets
}

\author{
SUN Rui-shan ${ }^{1, a}$, LI Shu-wen ${ }^{2, b^{*}}$ \\ ${ }^{1,2}$ Research Institute of Civil Aviation Safety, Civil Aviation University of China, Tianjin, China \\ asunrsh@hotmail.com \\ b*liishuwen@163.com
}

\begin{abstract}
In order to improve the neurobehavioral ability and personality characteristics of flight trainees and make them positively influence aviation safety by strengthening their learning during school. 39 undergraduate pilot students of the Class of 2019 were selected, their academic records were collected and they were tested for neurobehavioral ability. The results of perceptual ability, attentional ability and faculty of memory were obtained, and the data of personality characteristics were obtained through Eysenck Personality Questionnaire, training the neurobehavioral ability of pilot cadets, the relationship among the neurobehavioral ability, personality characteristics and academic performance was explored. The results indicate that memory was correlated with scores of aviation physiology, Introduction to Civil Aviation and flight operation and data $(\mathrm{P}<0.05)$, attention test was correlated with scores of flight system and power unit, pilot land-air communication, flight operation and data and other courses $(\mathrm{P}<0.05)$. There is no significant difference in introversion-extroversion between the pilot cadets' neurobehavioral ability and academic performance $(\mathrm{P}>0.05)$; the attention test results are significantly different from the results of neuroticism and psychoticism $(\mathrm{P}<0.05)$. The faculty of memory, attentional ability and neuroticism play an important role in the comprehensive ability and future development of pilot cadets. Attention is the focus of long - term training and strengthening of flight cadets.
\end{abstract}

Keywords: Pilot cadets, Academic performance, Neurobehavioral ability, Personality characteristics.

\section{飞行学员成绩、神经行为能力和人格特征之间的关系 研究}

\author{
孙瑞山 $1, \mathrm{a}$ 李淑雯 $2, \mathrm{~b}^{*}$
}

1,2 民航安全科学研究所, 中国民航大学, 天津，中国

asunrsh@hotmail.com

b*liishuwen@163.com

\section{摘要}

为加强飞行学员在校期间课程学习, 改造学员神经行为能力和人格特征, 使其正向影响航空安全。收集某大学 39 名 2019 级本科飞行学员的课业学习成绩、并进行神经行为能力测试, 得到关于感知觉、注意力和记忆的数 据, 通过艾森克人格检测得到人格特征数据, 进行综合分析, 探究飞行学员神经行为能力、人格特征和学业成 绩之间的关系。结果表明：记忆力与航空生理学、民航概论和飞行运行与资料等成绩显著相关 $(\mathrm{P}<0.05)$, 注 意力的测试结果与飞行系统与动力装置、飞行员陆空通话、飞行运行与资料等课程成绩显著相关 $(\mathrm{P}<0.05)$; 飞行学员的内外向程度与神经行为能力和学习成绩均无显著关系 ( $P>0.05)$, 飞行学员注意力测试结果与其自 身的情绪稳定性和气质类型有显著差异 $(\mathrm{P}<0.05)$ 。记忆力、注意力和情绪稳定性对飞行学员的综合能力和日 后发展有重要作用, 注意力是对飞行学员长期培训和加强的重点。 
关键词: 飞行学员, 成绩, 神经行为能力, 人格特征

\section{1.前言}

飞行职业的特殊性, 决定了除飞行技能的提高, 飞行学员的神经行为能力的培养和人格特征是教育 培训的关注重点。

飞行员的职业特性要求其具备良好的空间知觉、 运动知觉、记忆力和注意力, 这些能力综合体现为个 体的神经行为能力。在执行飞行任务的过程中, 飞行 员的神经行为能力对绩效产生影响 ${ }^{[1-2]}$ 。2009 年, 杨 仕云等 ${ }^{[3]}$ 指出, 民航招飞选拔要重视飞行员的空间能 力。2020 年, 王燕青等 ${ }^{[4]}$ 通过陆空通话的模拟实验, 得出飞行员注意力和记忆对陆空通话绩效影响显著 的结论。2017 年, 韩杨等 ${ }^{[5]}$ 通过大学生和飞行学员的 能力对比发现, 飞行学员的空间感知能力、记忆能力 和注意力分配和稳定性显著优于大学生。大量研究表 明, 行为能力倾向测试 (如空间定向测试、心理运动 测试）可以显著预测初始训练中个体飞行表现差异 ${ }^{6-}$

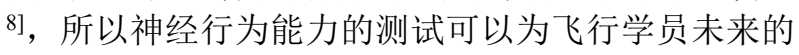
飞行综合素质评估提供帮助。

人格特征是影响个体心理健康的重要因素 ${ }^{[9]}$, 人 格特征形成后, 外部环境的影响很难使之发生变化, 因其具有自身独特性和稳定性 ${ }^{[10]}$ 且人格特征会影响 人的行为方式、处事风格和效率[11]。由于飞行职业的 特殊性, 飞行员人格特征一直备受关注。2003 年, Dillinger 等 ${ }^{[12]}$ 指出, 第一批关于人格的研究就集中在 评估适飞的军事航空飞行学员所应具备的人格特征, 2004 年, Fitzgibbons 等 ${ }^{[13]}$ 的调查发现飞行员的人格 特点是情绪稳定性高, 焦虑、脆弱、愤怒、冲动和抑 郁程度低, 2003 年, Bartram 等 ${ }^{[14]}$ 对英国陆军航空队 的研究表明, 训练合格的飞行员比那些训练失败的飞 行员更加稳定、外向、坚强和独立。2017 年, Ebru等 [15]根据对 24 名民航飞行学员训练成绩和所犯错误为 基础, 分析得出飞行学员在训练中所犯错误与人格特 征有密切关系。

本研究以飞行学员在校理论学习期间的成绩, 基 于神经行为测试评价系统 Neurobehavioral Evaluation System (NES-C3rd) 的神经行为能力测验数据和人格 特征为研究对象, 考察三者内在联系及其之间关系, 探究影响成绩和飞行的神经行为能力和人格特征。

\section{2. 研究方法}

\section{1. 研究对象}

从某民航院校 2019 级本科生中抽取 1 个班的飞 行学员, 剔除认知能力测试成绩超限、测试成绩有缺
失和在考试中有缺考、缓考者，保证数据有效、完整 的 39 名学员, 全部为男性。所学专业包括基础理论 类课程、飞行专业类课程和体能类课程。

\section{2. 测试工具}

使用艾森克人格检测（EPQ）对被试进行人格特 征测试, 飞行学员使用 NES-C3rd 进行 HF\&CRM 实 验, 得出关于感知觉能力、注意力和记忆相关的神经 行为能力测试成绩。

\section{3. 测试工内容}

\section{3. 1 , 成绩}

选用飞行学员在校学习成绩包括大学英语、飞行 员陆空通话、飞行英语 ESL、飞行系统与动力装置、 普通物理、航空生理学、高等数学、飞行运行资料、 民航概论、军事理论、体育等十余科。

\subsection{2，人格特征}

人格特征测试内容的三个维度为, $\mathrm{E}$ 维度: 内一 外向 (introversion-extroversion) 测试结果分为内向型、 中间型和外向型三种类型; $\mathrm{N}$ 维度: 情绪性, 也称神 经质（neuroticism）包括稳定性、中间型和不稳定型 三类特征, P 维度: 精神质 (psychoticism) 的人格特 征, 测试结果有胆汁质、多血-胆汁质、多血质、多种 气质类型混合型、多血-粘液质、粘液质六类。

\subsection{3. 神经行为能力}

知觉、记忆和注意力是神经行为能力的核心 [17], 进行的神经行为能力测试的内容包括感知觉能力、记 忆能力和注意能力三个部分。根据被反映事物的特性, 将感知觉能力分为形状知觉、运动知觉、空间知觉和 错觉。由此将感知觉实验分为以下四个部分: 形状知 觉 (SP), 反映对物体水平、垂直以及大小的感知能 力; 运动知觉 (MP), 主要反映对物体空间位移感知 能力; 空间知觉 (SS), 主要反映视感知-空间知觉能 力; 曲线吻合 ( $\mathrm{FC})$, 反映眼-手协调及非随意运动稳 定性。

注意将心理活动的各个过程紧密联系在一起, 是 心理活动的共同特性, 注意的品质包括注意的广度、 稳定性、注意力分配和注意力转移, 由此将注意实验 分为以下三个部分: 注意力分配 (DA), 反映视、听 感知-注意力分配和反应速度能力; 视注意广度 (AP), 
反映短时视记忆容量; 注意力调转 (SA), 反映视警 觉性、注意力以及反应的协调能力。

记忆是人脑对知识和过去经验的保存再现的心 理过程, 针对飞行特点, 将记忆实验分为以下四个部 分: 空间位置记忆广度 (SL), 反映视感知-空间位置 知觉能力; 记忆扫描 (MS), 反映短时记忆能力; 视
觉保留 (VR), 反映工作记忆和视觉记忆能力; 成对 词联想 (WA), 反映学习记忆-联想记忆能力。

\section{3. 结果}

\section{1. 成绩之间相关性}

分析各科成绩之间相关性如下表所示:

表 1 各科成绩之间的相关性

\begin{tabular}{|c|c|c|c|c|c|c|c|c|c|c|}
\hline \multirow{2}{*}{ 科目 } & \multicolumn{10}{|c|}{ 科目 } \\
\hline & 英语 & 系统 & 飞英 & 通话 & 数学 & 生理 & 军训 & 航概 & 体育 & 物理 \\
\hline 英语 & 1 & & & & & & & & & \\
\hline 系统 & 0.238 & 1 & & & & & & & & \\
\hline 飞英 & $0.672 * *$ & $0.322 *$ & 1 & & & & & & & \\
\hline 通话 & $0.454^{* *}$ & $0.574^{* *}$ & $0.534^{* *}$ & 1 & & & & & & \\
\hline 数学 & 0.192 & $0.542 * *$ & 0.230 & $0.493 * *$ & 1 & & & & & \\
\hline 生理 & 0.286 & $0.332 *$ & $0.395^{*}$ & $0.408^{*}$ & 0.263 & 1 & & & & \\
\hline 军训 & -0.069 & -0.002 & 0.067 & 0.305 & 0.092 & 0.017 & 1 & & & \\
\hline 航概 & 0.180 & $0.401 *$ & 0.237 & $0.367^{*}$ & 0.186 & 0.312 & -0.167 & 1 & & \\
\hline 体育 & -0.063 & 0.082 & 0.098 & 0.285 & 0.299 & 0.232 & $0.369^{*}$ & -0.130 & 1 & \\
\hline 物理 & 0.285 & $0.574^{* *}$ & $0.344 *$ & $0.661^{* *}$ & $0.782 * *$ & $0.407 *$ & $0.362 *$ & 0.166 & $0.470^{* *}$ & 1 \\
\hline
\end{tabular}

注: * $\mathrm{P}<0.05, * * \mathrm{P}<0.01$ 。系统 $=$ 飞行系统与动力装置, 飞英=飞行英语, 通话=飞行员陆空通话, 生理=航空生理学, 军理=军事理论, 航概 民航概论，运资=飞行运行与资料。下同

通过各科成绩相关性的对比结果可知, 此次调查 的飞行学员的英语成绩与飞行英语, 飞行员陆空通话 的成绩在 0.01 级别存在显著相关性, 体育成绩与军 训成绩在 0.05 级别存在显著相关性, 符合逻辑和实 际, 从表 1 可以看出, 除军训成绩和体育成绩外, 陆 空通话成绩与其他各科都存在显著相关性, 说明在飞 行学员在训练学习中, 陆空通话能力的提高是整体能
力的体现，陆空通话对职业发展和飞行安全有重要意 义, 是飞行学员未来培训的重点。

\section{2 神经行为能力之间的相关性}

对被试学员的神经行为能力成绩进行相关性分 析, 得到分析结果如下表所示:

\section{表 2 神经行为能力之间的相关性}

\begin{tabular}{|c|c|c|c|c|c|c|c|c|c|c|c|}
\hline 项 & \multicolumn{11}{|c|}{ 神经行为能力 } \\
\hline 目 & SP & MP & SS & SA & SL & $\mathrm{FC}$ & DA & $\mathrm{AP}$ & MS & WA & VR \\
\hline SP & 1 & & & & & & & & & & \\
\hline MP & 0.16 & 1 & & & & & & & & & \\
\hline SS & -0.10 & -0.13 & 1 & & & & & & & & \\
\hline SA & 0.14 & 0.107 & $0.52 * *$ & 1 & & & & & & & \\
\hline SL & -0.28 & -0.12 & 0.20 & -0.08 & 1 & & & & & & \\
\hline $\mathrm{FC}$ & -0.08 & -0.25 & 0.11 & -0.17 & 0.18 & 1 & & & & & \\
\hline DA & -0.14 & 0.02 & -0.16 & $-0.58 * *$ & 0.08 & -0.02 & 1 & & & & \\
\hline AP & $-0.35^{*}$ & -0.15 & -0.07 & $-0.16^{*}$ & 0.21 & -0.16 & $0.34^{*}$ & 1 & & & \\
\hline MS & -0.05 & -0.23 & -0.27 & $-.48 * *$ & 0.02 & -0.21 & $0.49^{* * *}$ & 0.24 & 1 & & \\
\hline WA & 0.00 & 0.08 & 0.27 & $0.46^{* *}$ & -0.03 & -0.21 & $-0.41^{*}$ & 0.11 & $-0.34 *$ & 1 & \\
\hline VR & 0.13 & -0.17 & -0.19 & $-.32 *$ & -0.03 & 0.03 & $0.40^{*}$ & $0.50 * *$ & 0.31 & -0.13 & 1 \\
\hline
\end{tabular}

由上表可以看出, 感知觉能力与记忆能力无相关 性, 记忆能力只与注意能力相关, 尤其是与注意力调
转和注意力分配关系密切。空间位置记忆广度与其他 神经行为能力都无关, 不受记忆、注意力和感知觉的 
影响。由此总结, 注意力与感知觉和记忆都有显著相 关性, 注意力的分配与调转在 0.01 级别显著相关。由 于个体注意力有限, 不能将注意力分配给过多的目标, 专注于重要的信息和操作项目, 避免临近的无关信息 源干扰是飞行学员在未来飞行中应具备的能力。

由表 2 得出的注意力影响范围广特性, 符合 Wickens 的信息处理模型 ${ }^{[16]}$, 该模型指出注意资源的 分配涉及飞行员执行任务过程中感知登记、知觉加工、 工作记忆、判断决策和响应执行的方面 ${ }^{[17]}$, 模型如图 1 所示。注意能力是飞行员在驾驶飞机过程中, 确保 飞行安全至关重要的能力, 也是飞行学员需要不断训 练的重点。

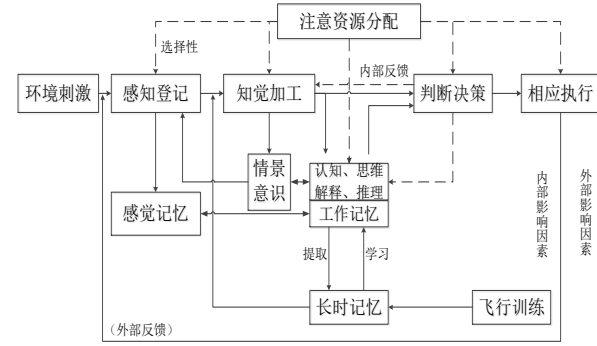

图 1 飞行员信息加工模型

\section{3. 神经行为能力与成绩关系}

将被试的各科成绩和神经行为能力成绩进行相 关性分析，得出结果如下表 3 所示:

表 3 神经行为能力和成绩之间的相关性

\begin{tabular}{|c|c|c|c|c|c|c|c|c|c|c|c|}
\hline \multirow{2}{*}{ 科目 } & \multicolumn{10}{|c|}{ 神经行为能力 } \\
\cline { 2 - 15 } & SP & MP & SS & SA & SL & FC & DA & AP & MS & WA & VR \\
\hline 英语 & -0.18 & 0.13 & -0.17 & 0.02 & $-0.44^{* *}$ & 0.12 & $-0.36^{*}$ & $0.47^{*}$ & $-0.37^{*}$ & -0.20 & $0.32^{*}$ \\
\hline 系统 & -0.02 & $-0.37^{*}$ & 0.03 & -0.05 & 0.17 & 0.04 & 0.12 & $0.34^{*}$ & 0.28 & 0.12 & -0.21 \\
\hline 飞英 & -0.14 & 0.19 & -0.10 & -0.06 & -0.11 & 0.21 & -0.24 & $0.35^{*}$ & 0.201 & -0.05 & -0.02 \\
\hline 通话 & 0.07 & -0.04 & -0.09 & -0.13 & 0.03 & $-0.35^{*}$ & -0.06 & 0.09 & 0.27 & -0.16 & 0.03 \\
\hline 数学 & -0.04 & -0.17 & 0.25 & $-0.32^{*}$ & 0.13 & $-0.33^{*}$ & 0.11 & $0.40^{*}$ & 0.13 & -0.01 & -0.07 \\
\hline 生理 & -0.02 & 0.03 & -0.05 & -0.13 & -0.03 & 0.01 & -0.14 & 0.14 & 0.07 & 0.03 & $0.32^{*}$ \\
\hline 军理 & 0.26 & -0.15 & 0.13 & -0.21 & -0.26 & $0.50^{* *}$ & -0.11 & 0.13 & 0.17 & 0.10 & $0.35^{*}$ \\
\hline 航概 & -0.05 & -0.25 & -0.04 & -0.01 & 0.17 & 0.11 & -0.03 & 0.07 & 0.10 & $-0.35^{*}$ & -0.05 \\
\hline 体育 & 0.17 & -0.08 & 0.05 & $-0.38^{*}$ & 0.05 & $0.66^{* *}$ & $0.34^{*}$ & -0.01 & -0.05 & 0.05 & 0.08 \\
\hline 物理 & 0.12 & 0.01 & 0.06 & -0.24 & 0.07 & -0.03 & 0.010 & $0.34 *$ & -0.15 & -0.13 & -0.05 \\
\hline 运资 & 0.03 & -0.00 & 0.21 & $0.36^{*}$ & $-0.35^{*}$ & 0.05 & -0.26 & $0.48^{* *}$ & $-0.36^{*}$ & 0.12 & $0.48^{*}$ \\
\hline
\end{tabular}

记忆保留、成对词联想、视觉保留和空间位置记 忆广度实验成绩与理论记忆类科目成绩相关 $(\mathrm{p}<0.05)$, 如英语、航空生理学、军事理论、民航概 论和飞行运行与资料, 这些课程考察学员的理解背诵 能力, 记忆能力与理论类科目成绩有相关性。曲线吻 合实验考察被试者的手眼协调性和非随意运动稳定 性, 其测试结果与体育成绩和军事理论成绩显著相关 $(p<0.01)$ 。除运动知觉与飞行系统与动力装置相关, 其他的感知觉能力与被试考试成绩均无相关性。

注意力测试成绩与理论概念类课程成绩无相关 性, 如航空生理学、军事理论、民航概论, 而这三门 课程主要考察记忆。其他课程都与注意力相关, 可见 注意力对于课程的学习是非常重要的。

\section{4. 人格特征与神经行为能力关系}

分别统计外向型、中间型和内向型飞行学员的神 经行为能力和成绩如表 4 所示:
表 4 按 $\mathrm{E}$ 维度统计结果

\begin{tabular}{|c|c|c|c|c|}
\hline \multirow{2}{*}{ 项目 } & \multicolumn{4}{|c|}{ 类型 } \\
\cline { 2 - 5 } & 外向型 & 中间型 & 内向型 & 差异性检 \\
& $x \pm s$ & $x \pm s$ & $x \pm s$ & 验 \\
\hline 英语 & $80.92 \pm 7.56$ & $78.09 \pm 9.83$ & $82.33 \pm 2.31$ & 0.566 \\
\hline 飞英 & $79.44 \pm 6.93$ & $75.91 \pm 8.75$ & $74.67 \pm 2.52$ & 0.301 \\
\hline 数学 & $81.56 \pm 7.47$ & $80.64 \pm 7.38$ & $88.33 \pm 5.77$ & 0.277 \\
\hline 通话 & $85.08 \pm 7.98$ & $74.64 \pm 9.90$ & $84.33 \pm 14.01$ & $0.010^{*}$ \\
\hline 运资 & $78.56 \pm 17.24$ & $81.36 \pm 5.43$ & $85.00 \pm 6.08$ & 0.706 \\
\hline 生理 & $74.96 \pm 7.70$ & $71.00 \pm 5.76$ & $72.00 \pm 4.58$ & 0.289 \\
\hline 军训 & $93.84 \pm 2.85$ & $91.91 \pm 3.14$ & $93.00 \pm 1.00$ & 0.190 \\
\hline 体育 & $77.40 \pm 10.64$ & $74.64 \pm 9.67$ & $68.67 \pm 2.08$ & 0.333 \\
\hline 军事 & $80.92 \pm 7.56$ & $78.09 \pm 9.83$ & $82.33 \pm 2.31$ & 0.566 \\
\hline 物理 & $80.16 \pm 11.91$ & $75.09 \pm 10.40$ & $86.00 \pm 7.55$ & 0.269 \\
\hline
\end{tabular}

由上表可以看出，个体的内向与否，与其自身的 感知觉能力、记忆力、注意力的好坏无明显差异, 与 学习成绩好坏也无明显差异。飞行学员的培训教育过 程中, 无需对学员的内外向性格多加干涉。 
根据艾森克人格检测得到情绪性测试结果，与飞 行学员的神经行为能力和成绩的统计结果如下表所 示:

表 5 按 $\mathrm{N}$ 维度统计结果

\begin{tabular}{|c|c|c|c|c|}
\hline \multirow{2}{*}{ 项目 } & \multicolumn{4}{|c|}{ 类型 } \\
\cline { 2 - 5 } & 不稳定型 $x \pm$ & 中间型 & 稳定型 & 差异性检 \\
& $s$ & $x \pm s$ & $x \pm s$ & 验 \\
\hline 英语 & $81.80 \pm 8.11$ & $80.83 \pm 9.03$ & $79.55 \pm 7.68$ & 0.817 \\
\hline 飞英 & $82.80 \pm 6.87$ & $76.67 \pm 8.16$ & $77.77 \pm 6.91$ & 0.289 \\
\hline 数学 & $82.60 \pm 5.86$ & $79.67 \pm 6.76$ & $82.82 \pm 8.09$ & 0.494 \\
\hline 通话 & $85.40 \pm 6.73$ & $79.58 \pm 9.75$ & $82.68 \pm 10.66$ & 0.510 \\
\hline 运资 & $64.80 \pm 36.44$ & $82.08 \pm 5.78$ & $82.05 \pm 5.65$ & $0.035^{*}$ \\
\hline
\end{tabular}

\begin{tabular}{|c|c|c|c|c|}
\hline 生理 & $73.80 \pm 4.76$ & $75.33 \pm 7.98$ & $72.64 \pm 7.16$ & 0.584 \\
\hline 军训 & $94.40 \pm 2.61$ & $91.83 \pm 3.90$ & $93.73 \pm 2.12$ & 0.123 \\
\hline 体育 & $86.20 \pm 6.06$ & $71.25 \pm 7.59$ & $76.18 \pm 10.50$ & $0.017^{*}$ \\
\hline 军事 & $83.80 \pm 10.06$ & $91.42 \pm 2.94$ & $91.00 \pm 2.16$ & $0.002^{* *}$ \\
\hline 物理 & $78.80 \pm 7.16$ & $74.00 \pm 10.52$ & $82.09 \pm 11.98$ & 0.142 \\
\hline
\end{tabular}

横向比较可得，被试的情绪稳定性与曲线吻合、 注意力调转、注意力分配的成绩有显著差异, 与其记 忆能力感知觉能力无显著差异。其中, 曲线吻合的测 试考察被试的非随意运动稳定性, 侧面反映了数据的 有效性。情绪稳定性与军事理论、飞行运行与资料和 体育成绩有显著差异，与其他课程无显著差异。

根据气质类型测试结果统计飞行学员的神经行 为能力和成绩如下表 6 所示:

\section{表 6 按 $\mathrm{P}$ 维度统计结果}

\begin{tabular}{|c|c|c|c|c|c|c|c|}
\hline \multirow[b]{2}{*}{ 项目 } & \multicolumn{7}{|c|}{ 类型 } \\
\hline & 胆汁质 $x \pm s$ & $\begin{array}{c}\text { 多血-胆汁质 } \\
x \pm s\end{array}$ & 多血质 $x \pm s$ & 混合型 $x \pm s$ & $\begin{array}{c}\text { 多血-粘液质 } \\
x \pm s\end{array}$ & 粘液质 $x \pm s$ & $\begin{array}{c}\text { 差异 } \\
\text { 性检 } \\
\text { 验 }\end{array}$ \\
\hline 军事 & $82.75 \pm 11.3$ & $90.83 \pm 3.71$ & $91.06 \pm 2.41$ & $91.80 \pm 2.28$ & $91.40 \pm 2.07$ & $89.67 \pm 0.58$ & $0.026^{*}$ \\
\hline 英语 & $80.00 \pm 8.12$ & $81.33 \pm 7.94$ & $81.50 \pm 7.81$ & $78.60 \pm 11.1$ & $75.40 \pm 8.96$ & $82.33 \pm 2.31$ & 0.761 \\
\hline 飞英 & $81.75 \pm 7.46$ & $76.00 \pm 5.93$ & $80.63 \pm 7.08$ & $75.40 \pm 10.5$ & $74.20 \pm 6.83$ & $74.67 \pm 2.52$ & 0.306 \\
\hline 数学 & $85.00 \pm 2.71$ & $81.00 \pm 5.87$ & $80.38 \pm 8.75$ & $79.20 \pm 8.47$ & $83.60 \pm 6.02$ & $88.33 \pm 5.77$ & 0.483 \\
\hline 通话 & $86.50 \pm 7.23$ & $84.33 \pm 4.55$ & $84.75 \pm 9.21$ & $72.80 \pm 11.7$ & $75.20 \pm 9.68$ & $84.33 \pm 14.0$ & 0.090 \\
\hline 运资 & $61.00 \pm 40.9$ & $83.17 \pm 5.49$ & $81.31 \pm 5.65$ & $79.40 \pm 5.59$ & $83.60 \pm 5.55$ & $85.00 \pm 6.08$ & 0.131 \\
\hline 生理 & $73.50 \pm 5.45$ & $79.00 \pm 7.69$ & $73.81 \pm 7.79$ & $70.20 \pm 6.69$ & $71.00 \pm 5.79$ & $72.00 \pm 4.58$ & 0.379 \\
\hline 军训 & $94.75 \pm 2.87$ & $92.33 \pm 3.98$ & $94.13 \pm 2.22$ & $91.00 \pm 4.53$ & $92.60 \pm 1.52$ & $93.00 \pm 1.00$ & 0.283 \\
\hline 体育 & $88.50 \pm 3.70$ & $73.00 \pm 8.07$ & $76.25 \pm 10.7$ & $69.00 \pm 8.12$ & $79.80 \pm 9.65$ & $68.67 \pm 2.08$ & $0.032 *$ \\
\hline 物理 & $80.25 \pm 7.37$ & $78.00 \pm 11.1$ & $80.50 \pm 13.3$ & $71.00 \pm 9.82$ & $79.60 \pm 11.2$ & $86.00 \pm 7.55$ & 0.579 \\
\hline
\end{tabular}

根据上表, 通过纵向对比可以看出, 个体的气质 类型对军事理论课程的影响大, 对飞行学员的体能状 态也影响较大, 此外, 被试的其他课程成绩无明显区 别。

\section{4. 结论}

本次实验结果表明:

(1) 注意力与感知觉能力和记忆力都相关, 是对 信息加工过程影响较大的神经行为能力, 与 Wickens 信息加工模型概念一致。飞行学员在学习和培训过程 中, 要不断锻炼提高其自身注意的分配能力、注意调 转能力, 这对未来进行飞机操纵、仪表观测、紧急情 况的处理有重要作用。

(2) 飞行学员的课程学习成绩与其自身感知觉 能力相关性不大, 理论性课程与记忆广度、记忆扫描、 视觉保留和成对词联想有关, 注意力广度、调转和分 配和对课程的学习影响较广, 良好的注意力调控对日
后学习工作有重要意义, 也是飞行学员在校课程学习 期间, 需要重点培训和加强的重点。

（3）个体的内外向程度与其成绩的好坏也无显 著关系。飞行学员稳定性程度是影响飞行安全的人格 特征因素, 在校课程学习时期关注和提高飞行学员的 情绪稳定性可以正向影响航空安全。

\section{REFERENCES}

[1] Ovaskainen H, Heikkila M. Visuospatial cognitive abilities in cut-to-length single-grip timber harvester work[J]. International Journal of Industrial Ergonomics, 2007, 37(9-10): 771-780.

[2] Komarraju, M, Karau S J, Schmeck R R, et al. The Big Five personality traits, learning styles and academic achievement[J]. Personality and individual differences, 2011, 51(4): 472-477.

[3] Yang Shiyun, Yan Bihua, You Xuqun.A Study on the 
Processing Level of Dynamic Spatial Ability in Civil Flight Pilots and Cadets[J]. Journal of Psychological Science, 2009, 32(01): 71-73+70.

[4] Wang Yanqing, Li Jia, Wang Yu. Research on Performance of Civil Aviation Pilots' Radiotelephony Communication Based on Cognitive Ability[J]. Science Technology and Engineering, 2020, 20(17): 7122-7128.

[5] Han Yang, Li Xiaojing,Ma Jin,et al. Comparative Study on Basic Cognitive Ability between Civil Pilot Students and College Students [J]. China Journal of Health Psychology, 2013, 21(03): 447449 .

[6] Burke, E. F., Hunter, D. R.. Meta-analysis of aircraft pilot selection procedures: Proceedings of the Seventh International Symposium on Aviation Psychology , Columbus: The Ohio State University, 413-417, 1993[C].

[7] Carretta, T. R., Ree, M. J.. Pilot-candidate selection method: Sources of validity $[\mathrm{J}]$. The International Journal of Aviation Psychology, 1994, 4(2): 103-117.

[8] Chidester, T. R., Helmreich, R. L., Gregorich, S. E.,et al. Pilot personality and crew coordination: Implications for training and selection[J]. The International Journal of Aviation Psychology, 1991, 1(1): 25-44

[9] FU Wenqing. Personality psychology[M]. Beijing: People's medical publishing House, 2007:168.

[10] McCrae RR , Costa PT. Nature over nurture: Temperament, personality, and life span development $[\mathrm{J}]$. Journal of Personality and Social Psychology, 2000, 178(1): 173-186.

[11] CHEN Zhonggeng, ZHANG Yuxin. Personality Psychology[M]. Shenyang : Liaoning People's Publishing House, 1986.

[12] Dillinger, T. G., Wiegmann, D. A., and Taneja, N.: Relating Personality with Stress Coping Strategies among Student Pilots in a Collegiate Flight Training Program, 12th International Symposium on Aviation Psychology, Dayton, 2003[C].

[13] Fitzgibbons, A., Schutte, P., and Davis, D. Pilot Personality Profile Using NEO-PI-R, National Aeronautics and Space Administration, 2004.

[14] Bartram, D. Personality Factors in Pilot Selection: Validation of theCathay Pacific Airways Selection Procedures, Proceedings of the Eight International Symposium on Aviation Psychology, Columbus, $\mathrm{OH}, 1330-1335[\mathrm{C}]$, The Ohio State University, 2003.

[15] Ebru Y. Fatma Canan, Doğan EROL. et,al. An
Analysis of the Factors Influencing Score Achieved during Pilot Training[J]. Transactions of the Japan Society for Aeronautical and Space Sciences, 2017, 60(4): 202-211.

[16] Wickens C D, Hollands J G. Engineering psychology and human performance[M]. New York: Harper-Collins. 1992.

[17] DU Hongbing, LIU Ming, JIN Huibin. Classification and Analysis of Pilots Errors Based on Information Processing Model [J]. Industrial Safety and Environmental Protection, 2013, 39(02): 90-93. 